\title{
Acute radiation enteritis causing small bowel obstruction
}

\author{
NJ Curtis, T Bryant, S Raj, AR Bateman, AH Mirnezami \\ Southampton University Hospitals NHS Trust, Southampton, UK
}

ABSTRACT

Although much is known about the late intestinal side effects of radiation, comparatively little has been published about its acute complications. We present a case of a small bowel obstruction due to acute radiation enteritis. As radiotherapy continues to expand its role in the management of oncological disease, clinicians should remain alert to the resulting undesired effects.

\section{KEYWORDS}

Radiation enteritis Radiotherapy Acute enteritis

Accepted 2 February 2011; published online 11 October 2011

\section{CORRESPONDENCE TO}

Nathan Curtis, Academic Clinical Fellow in General Surgical Oncology, University Surgical Unit, Level F Centre Block, Southampton University Hospitals NHS Trust, Tremona Road, Southampton S016 6YD, UK

T: +44(0)7719 137 249; E: nathancurtis@doctors.org.uk

\section{Case history}

A 77-year-old man with symptomatic sacral and pelvic side wall metastases from a prostatic endometrioid adenocarcinoma was started on high dose palliative radiotherapy. A 60Gy mid-plane dose was planned in thirty fractions over six weeks using an anterior to posterior field arrangement (Figs 1a and 1b) to encompass all known symptomatic metastases. After $40 \mathrm{~Gy}$ in 20 fractions over 28 days, he developed complete bowel obstruction. An examination revealed abdominal distension with diffuse tenderness and no abdominal scars. A raised white cell count of 14.4 x $10^{9} / 1$ and C-reactive protein of $213 \mathrm{mg} / \mathrm{l}$ were found. An abdominal $x$-ray revealed dilated loops of small bowel with a paucity of colonic gas. CT (Fig 1c) demonstrated several oedematous and thickened pelvic small bowel loops causing multifocal obstruction and a diagnosis of acute radiation enteritis (ARE) was made. After resuscitation, the patient was managed supportively with bowel rest and total parenteral nutrition.

Twelve days after the onset of symptoms, he suffered a self-limited but major lower gastrointestinal (GI) haemorrhage with a haemoglobin drop from $119 \mathrm{~g} / \mathrm{l}$ to $52 \mathrm{~g} / \mathrm{l}$. Emergency upper and lower GI endoscopies were conducted but these did not identify a cause in the upper tract or colorectum. After three weeks of conservative management, the patient's symptoms resolved with a gradual return of bowel activity and he was successfully discharged.

\section{Discussion}

Although much is known about the late intestinal side effects of radiation, comparatively little has been published about its acute complications. A search of the English language literature revealed no cases of acute bowel obstruction from ARE. ARE results from damage to the rapidly dividing mucosal crypt cells ${ }^{1}$ and microvascular endothelium. ${ }^{2}$ The degree of damage varies among individuals and is influenced by genomic susceptibility to ionising radiation. The patient described was not known to harbour any DNA damage repair defects. Acute non-obstructing small bowel injury is common in abdominopelvic radiotherapy. Although modern protocols with close attention to dose/fractionation, field size, arrangement and immobilisation techniques (eg use of a 'belly board' allowing displacement of the small bowel from the pelvis) can reduce the injury sustained, a dose of 30Gy or more can induce mucosal lesions in $90 \%$ of patients, with diarrhoea developing within two weeks. 1

The diagnosis of ARE is challenging and typically based on computed tomography appearances in the context of recent exposure. ${ }^{3}$ Mucosal lesions may also result in a clinically significant haemorrhage. ${ }^{5}$ Treatment is supportive as episodes are usually self limiting. Probiotics are of prophylactic use in reducing symptoms, particularly diarrhoea. ${ }^{1}$ High dose sulfasalazine can reduce the symptoms of ARE in established cases ${ }^{4}$ and loperamide has a role in reducing stool frequency and volume. ${ }^{2}$ Surgical intervention is considered a last resort and reserved for complicated mechanical obstruction and life threatening haemorrhage only. ${ }^{3}$ The irradiated tissue predisposes to an increased risk of fistulation and anastomotic leakage. For this reason, bypass or defunctioning stomas have been advocated. ${ }^{5}$ When necessary, resection and anastomosis using margins free from ARE are prudent and supported by studies on chronic radiation enteritis. ${ }^{5}$ As radiotherapy continues to expand its 
role in the management of oncological disease, clinicians should remain alert to the resulting undesired effects.

\section{References}

1. Zimmerer T, Böcker U, Wenz F, Singer MV. Medical prevention and treatment of acute and chronic radiation induced enteritis - is there any proven therapy? $A$ short review. Z Gastroenterol 2008; 46: 441-448.

2. Bismar MM, Sinicrope FA. Radiation enteritis. Curr Gastroenterol Rep 2002; 4: 361-365.

3. Toomey DP, Cahill RA, Geraghty J, Thirion P. Radiation enteropathy. Ir Med J 2006; 99: 215-217.

4. Kilic D, Ozenirler S, Egehan I, Dursun A. Sulfasalazine decreases acute gastrointestinal complications due to pelvic radiotherapy. Ann Pharmacother 2001; 35: 806-810.

5. Onodera H, Nagayama S, Mori A et al. Reappraisal of surgical treatment for radiation enteritis. World J Surg 2005; 29: 459-463.

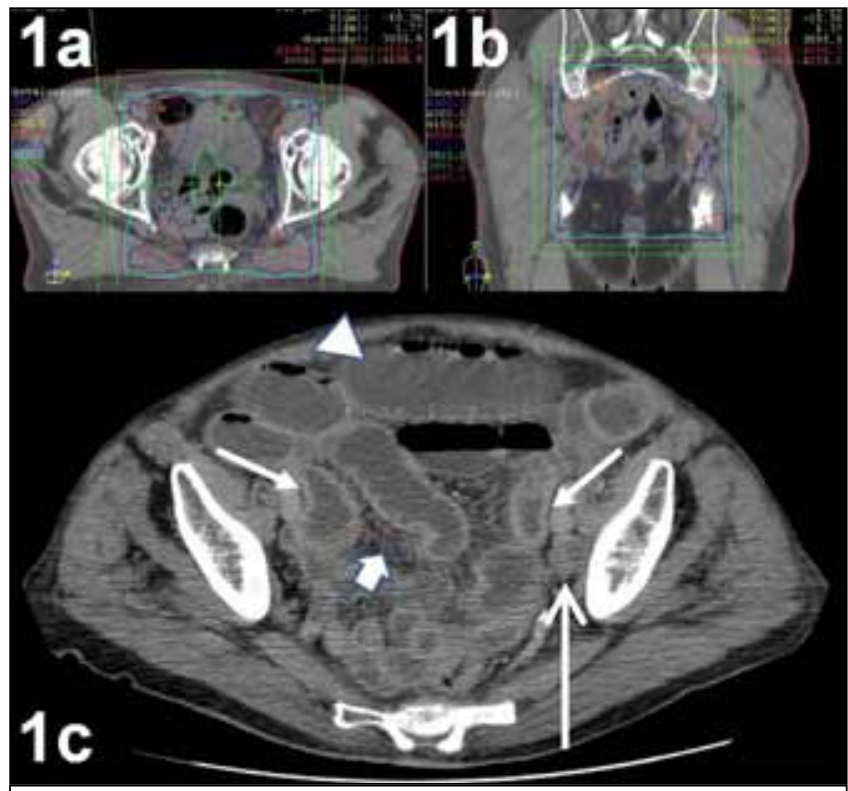

Figure 1 Axial (1a) and coronal (1b) views of radiotherapy fields with isodoses in cGy. Note the significant volume of small bowel in the anterior pelvis receiving 4,050 cGy (red isodose line). 1c: Portal venous phase axial CT image through the pelvis demonstrating small bowel wall thickening (closed arrows), mesenteric injection (block arrow) and proximal loop dilatation (arrowhead). Note the left pelvic side wall nodal recurrence (open arrow). 